Avrupa Bilim ve Teknoloji Dergisi

Özel Sayı 26, S. 73-77, Temmuz 2021

(C) Telif hakkı EJOSAT'a aittir

Araștırma Makalesi
European Journal of Science and Technology

Special Issue 26, pp. 73-77, July 2021

Copyright (C) 2021 EJOSAT

Research Article

\title{
Derin Öğrenme Tabanlı Mermer Yüzeylerinin Otomatik Sinıflandırılması
}

\author{
Mert Öktem, Şahin Alp Akosman, Özge Taylan Moral, Volkan Kılıç \\ İzmir Katip Çelebi Üniversitesi, Mühendislik ve Mimarlık Fakültesi, Elektrik-Elektronik Mühendisliği Bölümü, İzmir, Türkiye (ORCID: 0000-0002-0852-8681, 0000- \\ 0002-0261-7800, 0000-0003-0482-267X, 0000-0002-3164-1981),150403032@ ogr.ikcu.edu.tr, 160403042@ ogr.ikcu.edu.tr, ozgetaylan.moral@ikcu.edu.tr, \\ volkan.kilic@ikcu.edu.tr
}

(3rd International Congress on Human-Computer Interaction, Optimization and Robotic Applications June 11-13, 2021)

(DOI: $10.31590 /$ ejosat.950950)

ATIF/REFERENCE: Öktem, M., Akosman, Ş.A., Moral, Ö.T. \& Kılıç, V. (2021). Derin Öğrenme Tabanlı Mermer Yüzeylerinin Otomatik Sinıflandırılması. Avrupa Bilim ve Teknoloji Dergisi, (26), 73-77.

$\ddot{O} \mathbf{z}$

Doğal taşların mimaride ve dekorasyonda kullanımının artmasıyla mermere olan talep son yıllarda giderek yükselmiştir. Yükselen talebi karşılayabilmek için üreticilerin kapasite artıııı kadar, mermer üretim süreçlerinin verimliliğini de artırmaları gerekmektedir. Mermer üretim süreçlerinden biri olan mermer sınıflandırılmasında yapılan insan kaynaklı hatalardan dolayı üretim hızı ve verimi düşmektedir. $\mathrm{Bu}$ çalışmada, mermerlerin yanlış sınıflandırma problemine çözüm olarak farklı renk ve dokulara sahip mermer türlerinin yüksek başarımla sınıflandıran yapay zeka destekli bir sistem önerilmektedir. Önerilen sistemde, 5 farklı mermer türüne ait 516 mermer görüntüsünün sınıflandırılması için 12 evrişimsel sinir ağı mimarisi, transfer öğrenme ve derin öğrenme yöntemleri kullanılarak eğitilmiştir. Artırılmış veri kümesi ile yapılan eğitimler sonucunda transfer öğrenme uygulanan VGG-16 mimarisi ile \%96.07 sınıflandırma başarısı elde edilmiştir. Önerilen sistem, benzer çalışmalardan farklı olarak, geliştirdiğimiz arayüz ile birleştirilmiştir. Böylelikle, üretim sürecinde daha az insan kaynaklı hata ile mermerlerin sınıflandırılmasına katkı sağlanması amaçlanmıştır.

Anahtar Kelimeler: Mermer sınıflandırılması, Derin öğrenme, Transfer öğrenme, Evrişimsel sinir ağları.

\section{Deep Learning Based Automated Classification of Marble Surfaces}

\begin{abstract}
The demand for marble has increased gradually in recent years with the increasing use of natural stones in architecture and decoration. In order to meet the rising demand, producers must increase the efficiency of their marble production processes as well as increase their capacity. Production speed and efficiency decrease due to human-induced errors in marble classification, which is one of the marble production processes. In this study, as a solution to the problem of misclassification of marbles, an artificial intelligence supported system that classifies marble types with different colors and textures with high performance is proposed. In the proposed system, 12 convolutional neural network architectures were employed using transfer learning and deep learning methods to classify 516 marble images of 5 different marble types. As a result of the training with the augmented dataset, $96.07 \%$ classification accuracy was achieved with the VGG-16 architecture using transfer learning. Unlike similar studies, the proposed system has been integrated with our custom-designed interface. Thus, it is aimed to contribute to the classification of marbles which leads to less human-induced production issues.
\end{abstract}

Keywords: Marble classification, Deep learning, Transfer learning, Convolutional neural network.

*Sorumlu Yazar: 150403032@ogr.ikc.edu.tr 


\section{Giriş}

Mermer, estetik görünüşü ve sağlam yapısıyla iç ve dış cephe kaplamalarında yaygın olarak kullanılan dekoratif unsurlardan biridir. Mermer rezervi ve üretiminde büyük bir potansiyele sahip olan ülkemizde, mermer kullanımına olan talebin artmasıyla birlikte üretim kapasitesini artırabilecek, mevcut üretim hatalarını azaltan ve üretimi hızlandıran yeni çözümlere ihtiyaç duyulmaktadır. Mermerlerin üretim süreçlerinden biri olan mermerlerin sınıflandırılması, personel tarafindan gözle yapılmaktadır ki insan dikkat ve becerisine dayalı bu yöntem, üretim hızını ve verimini düşürmekle birlikte hata olasılığını da artırmaktadır (Cheung, 2006; Yin, Bai, \& Zhang, 2018).

Sınıflandırma problemine çözüm olarak görüntü işleme ve yapay zeka tabanlı otonom sistemler önerilmiştir. Segmentasyon ve kümeleme yöntemleri, mermer sınıflandırmada yaygın kullanılan yöntemlerdendir (Benavente \& Pina, 2009; KardanMoghaddama, Rajaeib, \& Moghaddam, 2018; Şişeci \& Çetişli, 2012). Görüntü işleme ve hiyerarşik kümelemeye dayalı bir algoritma ile konveyör sisteminde sınıflandırma yapılmıştır (Selver, Akay, Alim, Bardakçı, \& Ölmez, 2011). Başka bir çalışmada, mermer yüzeylerindeki renk dokusu analiz edilerek farklı renk uzaylarına göre öznitelikler çıkarılmıştır (MartínezAlajarín, Luis-Delgado, Tomás-Balibrea, \& Cybernetics, 2005). $\mathrm{Bu}$ öznitelikler, üç farklı katagoride sınıflandırma yapmak için 75 tane mermer örneği içeren veri kümesi üzerinde çok katmanlı algılayıcı sinir ağında eğitilmiştir (López, Martínez, Matías, Taboada, \& Vilán, 2010). Spektrofotometre kullanarak mermerlerden alınan spektral bilgi, fonksiyonel destek vektör makineleri ve fonksiyonel sinir ağı üzerinde eğitilmiştir. Mermer ve diğer yüzeyler üzerinde yapılan bu çalışmalarda, sınıflandırma yapılması için renk ve doku özniteliklerin çıkarılması gerekmektedir. Gerçek-zamanlı otonom sistemler çevresel koşullara açık olduğundan, öznitelik çıkarma işlemi sistemin verimliliğini ve doğruluğunu etkilemektedir.

Sınıflandırmanın başarılı olması görüntüden çıkarılan seçici özniteliklerine bağlıdır (Mercan \& Kılıç, 2020; Mercan, Kılıç, \& Şen, 2021; Mutlu \& Kılıç, 2018). Karmaşık öznitelikleri tespit etmek ve çevresel koşullardan etkilenme oranını minimize etmek için, son yıllarda sınıflandırma problemine derin öğrenme yaklaşımları uygulanmaktadır (Mercan, 2020; Mercan, Doğan, \& Kılıç, 2020). Derin öğrenme; nesne tanıma, konuşma tanıma, doğal dil işleme gibi alanlarda resim, video, ses ve metinlerden otomatik olarak öğrenebilen bir yapay zeka dalıdır (Umut, Yılmaz, \& Dikmen, 2019). Derin öğrenme geniş veri kümeleri ile öğrenme gerçekleştirir ve manuel özelik çıkarma işlemine gerek duymamaktadır (Buduma \& Locascio, 2017). Ayrıca, derin öğrenmenin bir alt dalı olan evrişimsel sinir ağları (ESA) aynı büyüklükteki yapay sinir ağlarına göre daha az bağlantı ve daha düşük sayıda eğitim parametresine sahiptir. Derin öğrenme tabanlı granit sınıflandırma çalışmasında MNIST1, MNIST2, MNIST3 ve CIFAR ESA'ları kullanılarak transfer öğrenme uygulanmış ve renkli karo görselleri üzerinde yüksek bir sınıflandırma doğruluğu elde edilmiştir (Ferreira \& Giraldi, 2017). Kendi geliştirdikleri ESA ile iki kalite sınıfından oluşan ve 80 mermer görüntüsü içeren veri kümesi eğitilmiştir (Pençe \& Çeşmeli, 2019). Veri artırımı uygulanmayan bu veri kümesinde, üç farklı algoritma ile 5-katlı çapraz doğrulama uygulanmıştır. ESA mimarilerinin kullanıldığı diğer bir yöntem olan transfer öğrenme, eğitim başarısı bilinen bir veri kümesi (örn. ImageNet) ile eğitilmiş mimarilerden bilgi transferi gerçekleştirilmesi olarak tanımlanır (Fırıldak \& Talu, 2019). Transfer öğrenme yönteminde, daha önce büyük bir veri kümesi ile eğitilmiş ağ ağırlıkları alınır ve farklı bir veri kümesi üzerinde kullanılır. Veri kümesi küçük olan sinıflandırma problemlerinde transfer öğrenme ile ağın daha iyi öğrenmesini sağlanmaktadır (Pan \& Yang, 2009).

$\mathrm{Bu}$ çalışmada üretim hatalarından biri kabul edilen mermerlerin yanlış sınıflandırma problemine çözüm olarak farklı renk ve dokulara sahip mermerleri yüksek doğrulukla sınıflandıran bir sistem önerilmiştir. Veri artırımı uygulanan 5 farklı sınıftan oluşan veri kümesi, 12 farklı ESA mimarisi kullanılarak derin öğrenme ve transfer öğrenme yöntemleri ile eğitilmiştir. Transfer öğrenme yöntemi uygulanan VGG-16 mimarisi ile en yüksek başarım elde edilmiş ve bu yöntem kullanıcı dostu bir arayüz ile birleştirilmiştir. Bildirinin geri kalanı ise şu şekilde düzenlenmiştir: Bölüm 2'de, mermer sınıflandırılması için derin öğrenme ve transfer öğrenme detaylandırılmış, veri artırımı ve arayüz tasarımı yöntemleri açıklanmıştır. Bölüm 3'te veri kümesi ve deneysel sonuçlar verilmiştir. Bölüm 4'te ise vargılara değinilmiştir.

\section{2. Önerilen Yöntem}

$\mathrm{Bu}$ bölümde, derin ve transfer öğrenme yöntemlerinin teorik içeriği anlatılmıştır. Ayrıca, çalışmada kullanılan veri artırımı yöntemi ve tasarlanan arayüz açıklanmıştır.

\subsection{Derin Öğrenme}

ESA tabanlı derin öğrenme mimarileri, evrişim katmanı, havuzlama katmanı, aktivasyon katmanı, tam bağlı katman ve yumuşatma gibi kendine özgü görevleri olan ayrı katmanlardan oluşur. Evrişim katmanında evrişim işlemi gerçekleştiren filtreler girdi boyutlarına göre taranır ve öznitelik haritası çıkarılır. Öznitelik haritasının uzamsal boyutunu düşürmek için örnekleme yapan havuzlama katmanı kullanılır. Tam bağlı katman önceki katmanların sonucunda üretilen değerleri girdi olarak alır ve sınıf sayısı kadar çıktı üretir (Kurt, 2018). Bu çalışmada, derin öğrenme eğitimlerine başlamadan önce kullanılacak olan mimarilerin tam bağlı katmanı, veri kümesinin içerdiği mermer sınıf sayısına göre 5 olarak yeniden şekillendirilmiştir. Çalışmada kullanılması için yeni ESA mimarisi oluşturulmamış, ImageNet veri kümesi üzerinde önceden tanımlı 1000 görüntü sınıfını başarıyla sınıflandıran VGG-16, AlexNet, GoogLeNet gibi mimariler tercih edilmiştir. Derin öğrenme yöntemi uygulanırken, ImageNet veri kümesi ile eğitilip elde edilen katsayılar kullanılmamıştır. Mermer veri kümesi, mimariler kullanılarak eğitilmiş ve yeni katsayılar oluşturulmuştur.

\subsection{Transfer Öğrenme}

Derin öğrenmeden farklı olarak transfer öğrenme, görüntü özniteliklerini ağın üst katmanlarını kullanmadan çıkarır. $\mathrm{Bu}$ yöntemde, uygun olan katman (tam bağlı katman) bulunana kadar katmanlar dondurulur ve belli bir iterasyonda eğitildikten sonra yeni katmanlar eklenir. Daha önce büyük bir veri kümesi üzerinde eğitilmiş ağın katsayıları ve değiştirilmiş ağ yapısı ile mevcut veri kümesi sınıflandırılır. Transfer öğrenme eğitimleri, derin öğrenmede kullanılan 12 ön-tanımlı mimari ve katsayılarıyla gerçekleştirilmiştir. $\mathrm{Bu}$ katsayılar, ImageNet üzerinde bulunan 1000 görüntü sınıfinın ait 14 milyondan fazla görüntünün eğitilmesiyle belirlenmiştir. 


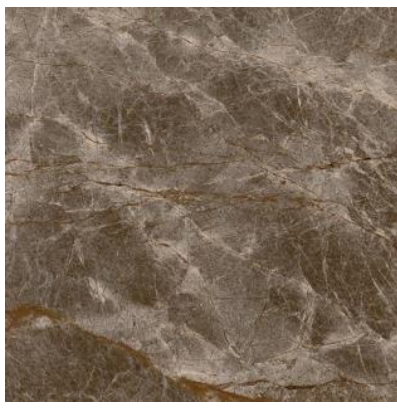

(a)
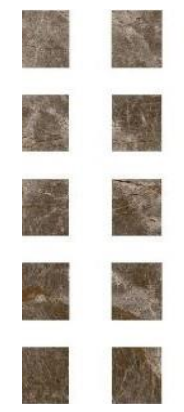

(b)

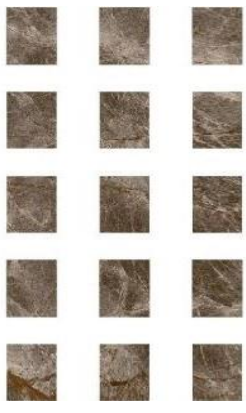

Şekil 1. EMPERADOR sinufina ait orijinal resim (a)'da gösterilmişken veri artırımı uygulanmış resim (b)'de verilmiştir.

\subsection{Veri Artırımı}

Derin öğrenme yöntemlerinde, veri kümesinin sahip olduğu içerik sayısı sınıflandırma performansında büyük rol oynamaktadır. Bu nedenle, içerik sayısı az olan veri kümelerinde veri artırımı yöntemi uygulanarak içerik sayısı artırılmakta, dolayısıyla eğitimlerin başarısı artmakla birlikte aşırı öğrenmenin (overfitting) önüne de geçilmektedir. Veri artırımı, veri kümesindeki görüntülerin döndürme, öteleme, kırpma, soldurma, ölçeklendirme gibi işlemlerden geçirerek sentetik kopyalarını oluşturma ve yeni görüntüleri mevcut veri kümesine ekleyerek büyük bir veri kümesi oluşturma işlemidir (Shorten \& Khoshgoftaar, 2019). Bu klasik veri artırımı yöntemleri, içerdiği şekil bilgisinden daha çok renk ve doku ile öne çıkan mermer yüzeylerinde etkili olmamaktadır. $\mathrm{Bu}$ nedenle, veri artırımı klasik yöntemlerin yerine yüksek çözünürlüklü ve boyuttaki mermer görüntülerinden, her bir ESA mimarisinin girdi boyutlarına uygun adaptif yeni görüntüler oluşturulmuştur.

Uygulanan veri artırımı yöntemi ile, bir mermer görüntüsü, kullanılacak mimarinin giriş çözünürlüğüne göre eşit parçalara bölünür. Görüntü boyutundan dolayı oluşan eşit olmayan parçalar, giriş çözünürlüğüne göre yeniden boyutlandırılır. Giriş çözünürlüğü 224x224 piksel olan VGG-16 mimarisi için veri artırımı uygulanmış EMPERADOR sınıfına ait 1024x1024 piksel çözünürlüğündeki bir mermer görüntüsü Şekil 1'de gösterilmiştir. $\mathrm{Bu}$ çalışmada kullanılan ESA mimarileri farklı giriş çözünürlüklerine sahiptir. Mimarilerin en düşük giriş çözünürlüğü 224x224 piksel iken en yüksek giriş çözünürlüğü $331 \times 331$ pikseldir. $\mathrm{Bu}$ nedenle, orijinal veri kümesi, giriş çözünürlüğü en küçük 224x224 piksel olan mimari için 8394 görüntü oluşturulurken, giriş çözünürlüğü en yüksek 331x331 piksel olan mimari için 3874 görüntü içeren veri kümesi oluşturulabilmiştir. Diğer mimarilerden oluşan veri kümeleri ise 3874-8394 arasında görüntü içermektedir.

\subsection{Arayüz Tasarımı}

Mermer sınıflandırma işleminin kolay ve hızlıca yapılabilmesi için MATLAB AppDesigner kullanarak tasarladığımız arayüz, en yüksek başarımı sağlayan sınıflandırıcı ile birleştirilmiştir. Şekil 2'de kullanıcı arayüzünün ekran görüntüsü verilmiştir. Arayüzde sınıflandırma yapılması için 3 seçenek bulunmaktadır. Kullanıcı "Tekli Sınıflandır" seçeneğine tıkladığında, seçilen mermer görüntüsü, mermerin hangi sınıfa ait olduğunu gösteren pasta grafiği ve sinıflandırma doğruluk yüzdesi ekranda görülür.

e-ISSN : 2148-2683

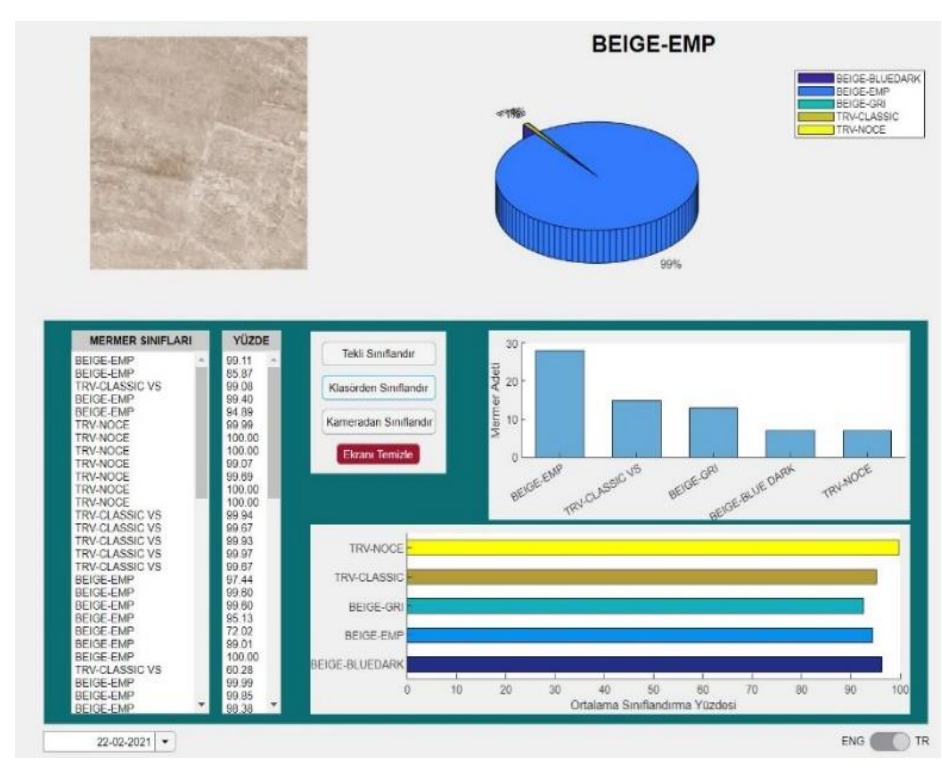

Şekil 2. Sinıflandırma arayüzü

Kullanıcı "Klasörden Sınıflandır" seçeneğine tıkladığında, seçilen klasördeki tüm mermer görüntüleri sırasıyla sınıflandırılır ve ilgili mermer görüntüsüne ait doğruluk yüzdesi pasta grafiği üzerinde gösterilir. Sinıflandırılan mermer görüntüleri otomatik olarak ilgili klasöre kaydedilir. Klasörlerde bulunan mermer adedi ve ortalama sınıflandırma yüzdesi tablolarda verilir. Kullanıcı, "Kameradan Sınıflandır" seçeneğine tıkladığında, kamera ile yakalanan görüntü üzerinde sınıflandırma yapılır. Ayrıca, kullanıcı geçmiş sınıflandırma sonuçlarına görmek isterse tarih seçerek ilgili klasöre ulaşabilir. Sınıflandırma yapılmamış bir tarih seçildiğinde, kullanıcı uyarı mesajı ile uyarılır. İngilizce ve Türkçe dil desteğiyle gelen arayüzümüz üretim hattında çalışanların rahatlıkla kullanabileceği basit ve sade bir tasarıma sahiptir.

\section{Deneysel Sonuçlar}

\subsection{Veri Kümesi}

Eğitimlerde kullanılan veri kümesi 5 mermer sınıfına ait 516 mermer görüntüsü içermektedir. Bu görüntülerden 102 tanesi BEIGE-GRI, 90 tanesi BEIGE-BLUE DARK, 42 tanesi BEIGEEMPERADOR, 91 tanesi TRAVERTINE-NOCE ve 191 tanesi ise TRAVERTINE-CLASSIC sınıfına aittir. Veri kümesinin türlerine göre içerdiği örnek mermer görüntüleri Şekil 3'te gösterilmiştir.

Eğitimlerde kullanılmak üzere her mermer sınıfı için, içerdiği görüntülerin \%30'u rastgele seçilerek test veri kümesi oluşturulmuş ve \%70'i ise eğitim veri kümesi olarak kullanılmıştır. Böylece aşırı öğrenmenin önüne geçilmesi hedeflenmiştir. Görüntülerin 361 tanesi eğitim kümesi olarak, 154 tanesi ise test kümesi olarak gruplandırılmıştır. Bu görüntü sayıları, derin ve transfer öğrenme mimarilerinin eğitimi için yeterli olmadığından, görüntüler Bölüm 2.3'te açıklanan veri artırımı yöntemi ile mimarinin giriş çözünürlüğüne göre adaptif olarak çoğaltılmıştır. 


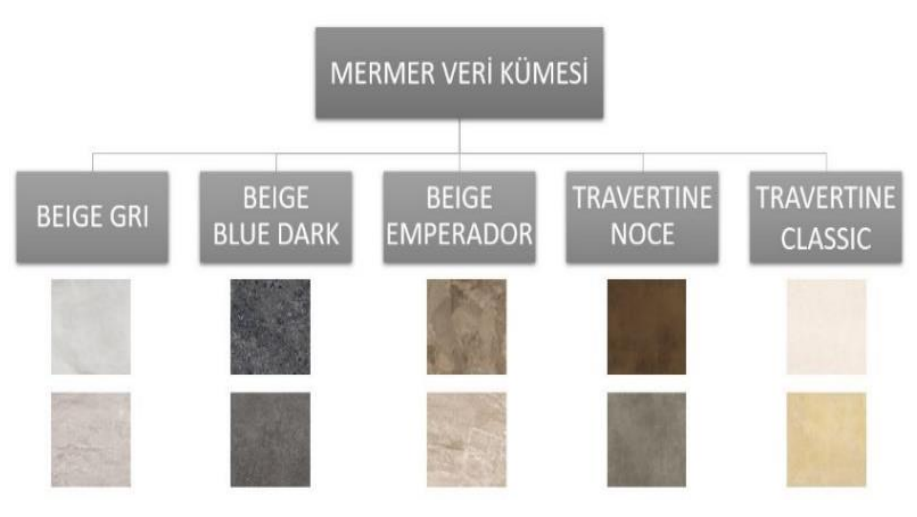

Şekil 3. Veri kümesi

\subsection{Sonuçlar}

$\mathrm{Bu}$ çalışmada, veri kümesi belirlenen 12 farklı ESA mimarisi ile derin öğrenme ve transfer öğrenme yöntemleriyle eğitilmiştir. Her bir mimari için veri kümesi, giriş çözünürlüklerine göre yeniden boyutlandırılmıştır. Derin öğrenme ve transfer öğrenme yöntemlerinde parametreler sabit tutulmuştur. Mimariler eğitilirken, daha önceden belirlenen hiper parametre değerleri, eğitim tur sayısı 20 ve mini-batch 16 olacak şekilde ayarlanmıştır. Orijinal ve veri artırımı yöntemi ile oluşturulan yeni veri kümeleri üzerinde eğitimler aynı mimariler referans alınarak tekrarlanmıştır.

Derin öğrenme ve transfer öğrenme yöntemleri ile gerçekleştirilen eğitimlerin sonuçları Tablo 1'de verilmiştir. Derin öğrenme ile eğitilen orijinal veri kümesi üzerinde en yüksek doğruluk yüzdesi \%78.78 ile GoogLeNet mimarisinde elde edilirken artırılmış veri kümesi ile yapılan eğitimler sonucunda en yüksek doğruluk yüzdesi \%88.80 ile VGG-16 mimarisinde görülmüştür. Transfer öğrenme eğitimleri sırasında derin öğrenme yönteminden farklı olarak mimarilerin katsayıları kullanılmıştır. Orijinal veri kümesiyle gerçekleştirilen transfer öğrenme eğitimlerinde VGG-16 ve VGG-19 mimarilerinden $\% 85.81$ ile en iyi doğruluk yüzdesi elde edilirken artırılmış veri kümesi ile yapılan eğitimler sonucunda en yüksek doğruluk yüzdesi \%96.07 ile VGG-16 mimarisinde gözlemlenmiş ve bu eğitime ait hata matrisi (veya karmaşıklık matrisi) Şekil 4'te verilmiştir.

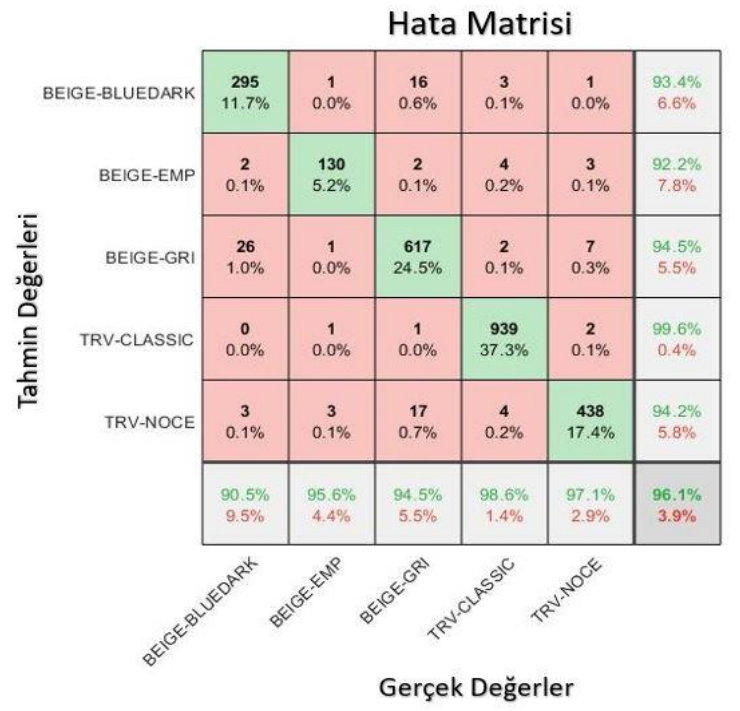

Şekil 4. Artırılmış veri kümesi ile transfer öğrenme yapılan $V G G-16$ mimarisine ait hata matrisi
Tablo 1. Derin ve Transfer Öğrenme Doğruluk Yüzdeleri

\begin{tabular}{|c|c|c|c|c|}
\hline \multirow[b]{2}{*}{ ESA } & \multicolumn{2}{|c|}{$\begin{array}{c}\text { DERİN } \\
\text { ÖĞRENME }\end{array}$} & \multicolumn{2}{|c|}{$\begin{array}{l}\text { TRANSFER } \\
\text { ÖĞRENME }\end{array}$} \\
\hline & \begin{tabular}{|c|} 
Orijinal \\
Veri \\
Kümesi \\
$(\%)$
\end{tabular} & $\begin{array}{l}\text { Artırılmış } \\
\text { Veri } \\
\text { Kümesi } \\
(\%)\end{array}$ & $\begin{array}{c}\text { Orijinal } \\
\text { Veri } \\
\text { Kümesi } \\
(\%)\end{array}$ & $\begin{array}{c}\text { Artırılmış } \\
\text { Veri } \\
\text { Kümesi } \\
(\%)\end{array}$ \\
\hline Inception-v3 & 71.61 & 69.07 & 82.58 & 78.72 \\
\hline Xception & 77.74 & 80.64 & 83.87 & 85.21 \\
\hline DarkNet-53 & 75.48 & 83.79 & 81.29 & 86.45 \\
\hline MobileNet-v2 & 70.97 & 83.60 & 78.71 & 90.03 \\
\hline DarkNet-19 & 78.06 & 81.59 & 76.13 & 91.07 \\
\hline ResNet-101 & 67.74 & 78.16 & 84.52 & 92.02 \\
\hline ResNet-50 & 72.26 & 78.99 & 82.58 & 92.34 \\
\hline ResNet-18 & 78.71 & 86.06 & 82.58 & 92.37 \\
\hline AlexNet & 78.06 & 87.88 & 82.58 & 93.37 \\
\hline GoogLeNet & 78.78 & 88.32 & 81.94 & 94.44 \\
\hline$V G G-19$ & 77.42 & 88.64 & 85.81 & 95.87 \\
\hline$V G G-16$ & 78.05 & 88.80 & 85.81 & 96.07 \\
\hline
\end{tabular}

Hata matrisi test veri kümesinin gerçek etiketleri ve eğitim sonunda tahmin edilen tahmin etiketlerinden oluşur. Hata matrisinin sütunları gerçek etiketlere, satırları tahmin edilen etiketlere karşılık gelir. Köşegen hücreler (yeşil renkli) doğru sınıflandırılmış etiketleri belirtirken köşegen olmayan her hücre (kırmızı renkli) yanlış sınıflandırılmış etiketleri temsil eder. Yapılan eğitimlerde ESA mimarilerinin giriş çözünürlüklerine göre genişletilen veri kümelerinin, derin öğrenme ve transfer öğrenme eğitimlerinde başarım oranını yükselttiği görülmüştür. Tablo 2'de önerilen yöntem ve diğer mermer sınıflandırma çalışmalarının kıyaslaması verilmiştir. Sonuçlar önerilen yöntemin üstünlüğünü göstermektedir.

Tablo 2. Mermer Sınıflandırma Çalışmaları Sonuçları

\begin{tabular}{||c||c|c||}
\hline \hline & YÖNTEM & DOĞRULUK (\%) \\
\hline \hline (Pençe \& Çeşmeli, 2019) & ESA & 75.00 \\
\hline \hline $\begin{array}{c}\text { (KardanMoghaddama et al., } \\
\text { 2018) }\end{array}$ & $\begin{array}{c}\text { K-means } \\
\text { Kümeleme }\end{array}$ & 83.60 \\
\hline \hline (Selver et al., 2011) & $\begin{array}{c}\text { Hiyerarşik } \\
\text { Kümeleme }\end{array}$ & 83.60 \\
\hline \hline (Ferreira \& Giraldi, 2017) & CIFAR & 87.26 \\
\hline \hline Önerilen Yöntem & VGG-16 & $\mathbf{9 6 . 0 7}$ \\
\hline \hline
\end{tabular}

\section{Vargilar}

$\mathrm{Bu}$ çalışmada, mermerlerin üretim süreçlerinden yanlış sınıflandırma problemine çözüm olarak derin öğrenmeye dayalı bir yöntem önerilmiştir. Mermer yüzeyleri içerdikleri şekil bilgisinden daha çok renk ve doku ile öne çıktıkları için klasik veri artırım yöntemlerinden farklı olarak her bir ESA mimarisi giriş çözünürlüğüne uygun adaptif yeniden boyutlandırma yöntemi uygulanmıştır. Çalışmada 5 farklı veri kümesine ait orijinal ve adaptif oluşturulmuş yeni veri kümeleri derin öğrenme ve transfer öğrenme yöntemleri ile eğitilmiştir. Eğitimler sonucunda, genişletilen veri kümesinin eğitim başarısını artırdığı gözlemlenmiştir. Artırılmış veri kümesi ile VGG-16 mimarisinde \%96.07 sınıflandırma başarısı elde edilmiştir. Ayrıca, üretim sürecinde kullanıma ve insan kaynaklı 
hataları azaltmaya yönelik basit ve sade tasarımı olan İngilizceTürkçe dil desteğine sahip, kullanıcının tercihine göre sınıflandırma seçenekleri sunan ve geçmişe yönelik kayıt tutan bir arayüz tasarlanmıştır.

\section{Teşekkür}

Bu çalışma, TÜBİTAK 2209-B Sanayiye Yönelik Lisans Araştırma Projeleri Desteği Programı (Proje no: 1139B412000853) ve İzmir Katip Çelebi Üniversitesi Bilimsel Araştırma Projeleri Koordinatörlüğü (Proje no: 2019-GAPMÜMF-0013) tarafından desteklenmektedir.

\section{Kaynakça}

Benavente, N., \& Pina, P. (2009). Morphological segmentation and classification of marble textures at macroscopical scale. J Computers geosciences, 35(6), 1194-1204.

Buduma, N., \& Locascio, N. (2017). Fundamentals of deep learning: Designing next-generation machine intelligence algorithms: " O'Reilly Media, Inc.".

Cheung, K. S. (2006). Modelling and analysis of manufacturing systems using augmented marked graphs. J Information Technology Control, 35(1).

Ferreira, A., \& Giraldi, G. (2017). Convolutional Neural Network approaches to granite tiles classification. $J$ Expert Systems with Applications, 84, 1-11.

Fırıldak, K., \& Talu, M. F. (2019). Evrișimsel Sinir Ağlarında Kullanılan Transfer Öğrenme Yaklaşımlarının İncelenmesi. J Bilgisayar Bilimleri, 4(2), 88-95.

KardanMoghaddama, H., Rajaeib, A., \& Moghaddam, H. K. (2018). Marble slabs classification system based on image processing (ark marble mine in Birjand). J Civil Engineering Journal, 4(1).

Kurt, F. (2018). Evrişimli Sinir A ğlarında Hiper Parametrelerin Etkisinin İncelenmesi. Hacettepe Üniversitesi Fen Bilimleri Enstitüsü,

López, M., Martínez, J., Matías, J. M., Taboada, J., \& Vilán, J. A. (2010). Functional classification of ornamental stone using machine learning techniques. Journal of Computational Applied Mathematics, 234(4), 13381345.

Martínez-Alajarín, J., Luis-Delgado, J. D., Tomás-Balibrea, L.M. J. I. T. o. S., Man,, \& Cybernetics, P. C. (2005). Automatic system for quality-based classification of marble textures. 35(4), 488-497.

Mercan, Ö. B. (2020). Deep Learning based Colorimetric Classification of Glucose with Au-Ag nanoparticles using Smartphone. Paper presented at the 2020 Medical Technologies Congress (TIPTEKNO).

Mercan, Ö. B., Doğan, V., \& Kılıç, V. (2020). Time Series Analysis based Machine Learning Classification for Blood Sugar Levels. Paper presented at the 2020 Medical Technologies Congress (TIPTEKNO).

Mercan, Ö. B., \& Kılıç, V. (2020). Fuzzy classifier based colorimetric quantification using a smartphone. Paper presented at the International Conference on Intelligent and Fuzzy Systems.

Mercan, Ö. B., Kılıç, V., \& Şen, M. (2021). Machine learningbased colorimetric determination of glucose in artificial saliva with different reagents using a smartphone coupled $\mu$ PAD. J Sensors Actuators B: Chemical, 329, 129037.
Mutlu, A. Y., \& Kılıç, V. (2018). Machine learning based smartphone spectrometer for harmful dyes detection in water. Paper presented at the 2018 26th Signal Processing and Communications Applications Conference (SIU).

Pan, S. J., \& Yang, Q. (2009). A survey on transfer learning. IEEE Transactions on Knowledge Data Engineering, 22(10), 1345-1359.

Pençe, İ., \& Çeşmeli, M. Ş. (2019). Deep learning in marble slabs classification. J Scientific Journal of Mehmet Akif Ersoy University, 2(1), 21-26.

Selver, M. A., Akay, O., Alim, F., Bardakçı, S., \& Ölmez, M. (2011). An automated industrial conveyor belt system using image processing and hierarchical clustering for classifying marble slabs. $J$ Robotics ComputerIntegrated Manufacturing, 27(1), 164-176.

Shorten, C., \& Khoshgoftaar, T. M. (2019). A survey on image data augmentation for deep learning. J Journal of Big Data, 6(1), 1-48.

Şişeci, M., \& Çetişli, B. (2012). Traverten plaka taşlarda sinifların K-ortalamalar ve bulanık C-ortalamalar kümeleme yöntemleri ile belirlenmesi. J Süleyman Demirel Üniversitesi Fen Bilimleri Enstitüsü Dergisi, 16(3), 238-247.

Umut, K., Yılmaz, A., \& Dikmen, Y. (2019). Sağlık alanında kullanılan derin öğrenme yöntemleri. J Avrupa Bilim ve Teknoloji Dergisi(16), 792-808.

Yin, J.-f., Bai, Q., \& Zhang, B. (2018). Methods for detection of subsurface damage: a review. J Chinese Journal of Mechanical Engineering, 31(1), 1-14. 